Article

\title{
The Design and Optimization of Plasmonic Crystals for Surface Enhanced Raman Spectroscopy Using the Finite Difference Time Domain Method
}

\author{
Alec Bigness and Jason Montgomery * \\ Department of Chemistry, Biochemistry, and Physics, Florida Southern College, Lakeland, FL 33801, USA; \\ abigness@flsouthern.edu \\ * Correspondence: jmontgomery@flsouthern.edu
}

Received: 30 March 2018; Accepted: 24 April 2018; Published: 26 April 2018

\begin{abstract}
We present computational studies of quasi three-dimensional nanowell (NW) and nanopost (NP) plasmonic crystals for applications in surface enhanced Raman spectroscopy (SERS). The NW and NP plasmonic crystals are metal coated arrays of cylindrical voids or posts, respectively, in a dielectric substrate characterized by a well/post diameter $(D)$, relief depth $(R D)$, periodicity $(P)$, and metal thickness $(M T)$. Each plasmonic crystal is modeled using the three-dimensional finite-difference time-domain (FDTD) method with periodic boundary conditions in the $x$ - and $y$-directions applied to a computational unit cell to simulate the effect of a periodic array. Relative SERS responses are calculated from time-averaged electric field intensity enhancements at $\lambda_{\text {exc }}$ and $\lambda_{\text {scat }}$ or at $\lambda_{\text {mid }}$ via $G_{\text {SERS }}^{4}=g^{2}\left(\lambda_{\text {exc }}\right) \times g^{2}\left(\lambda_{\text {scat }}\right)$ or $G_{\text {mid }}^{4}=g^{4}\left(\lambda_{\text {mid }}\right)$, respectively, where $g^{2}=|E|^{2} /\left|E_{0}\right|^{2}$. Comparisons of $G_{\text {SERS }}^{4}$ and $G_{\text {mid }}^{4}$ are made to previously reported experimental SERS measurements for NW and NP geometries. Optimized NW and NP configurations based on variations of $D, P, R D$, and $M T$ using $G_{\text {SERS }}^{4}$ are presented, with $6 \times$ and $2 \times$ predicted increases in SERS, respectively. A novel plasmonic crystal based on square NP geometries are considered with an additional $3 \times$ increase over the optimized cylindrical NP geometry. NW geometries with imbedded spherical gold nanoparticles are considered, with $10 \times$ to $10^{3} \times$ increases in SERS responses over the NW geometry alone. The results promote the use of FDTD as a viable in silico route to the design and optimization of SERS active devices.
\end{abstract}

Keywords: finite-difference time-domain; FDTD; surface plasmons; surface enhanced Raman spectroscopy; soft nanolithography

\section{Introduction}

Surface enhanced Raman spectroscopy (SERS) employing metallic nanostructures is a well studied phenomenon that arises predominantly from large electric field intensity enhancements when light is incident on a metal that supports surface plasmons (SPs) [1-3]. SPs are collective oscillations of conducting electrons with a characteristic frequency that can exist at the interface of a dielectric and a metal $[4,5]$. Under certain conditions, incident light resonant with the plasmon frequency can excite the SP within the metal, giving rise to sub-wavelength field confinement and intensity enhancements, defined hereafter as $g^{2}=|E|^{2} /\left|E_{0}\right|^{2}$, where $|E|$ and $\left|E_{0}\right|$ are the magnitudes of the total and incident electric field, respectively. (The spatial and frequency/wavelength dependence of $E$ is ignored for the moment for simplicity.) The SP frequency is influenced by the size, shape, and local dielectric environment of the metal and can thereby be tuned as desired [6,7]. Molecules that traverse the SP field experience not only enhanced absorption of light proportional to the enhancement factor at the excitation frequency, $g_{\text {exc }}^{2}$, but also enhanced Raman scattering proportional to the enhancement factor at the Raman scattered frequency, $g_{\text {scat }}^{2}$. The overall SERS response is then proportional to $g_{\mathrm{exc}}^{2} \times g_{\text {scat }}^{2}[8]$. Early SERS substrates 
were composed of rough metal surfaces or colloidal solutions of metallic nanoparticles whose sensitivity and reproducibility suffered from the generation of only random "hot-spots" on the metal surface or in the temporal gaps between adjacent particles. Within the last decade, nanolithography methods based on soft imprinting have enabled the inexpensive fabrication of robust SERS substrates that give rise to uniform hot-spots over large areas, with enhancement factors on the order of $10^{6}$ to $10^{8}$ [9-12].

The SERS substrates considered in this work are based on quasi-three-dimensional plasmonic crystals composed of dielectric supports patterned with either voids or posts upon which thin films of gold are deposited using electron beam evaporation. We do not consider sputtering, which can give rise to wall features. Nanowell (NW) geometries (Figure 1a) result from voids in the supporting dielectric [13], and nanopost (NP) geometries (Figure 1b) result from posts on the supporting dielectric [14]. Previous experimental studies of these NW and NP geometries involved fabricating an array of plasmonic crystals, each region of the array characterized by a particular diameter, $D$, and periodicity, $P$. The SERS response of a target molecule, namely benzene thiol, was measured for each region of the array. A finite-difference time-domain (FDTD) analysis of the geometries studied showed qualitative agreement (more on this below) between relative SERS responses, and a maximum in the SERS response was attributed to the excitation of a local surface plasmon resonance (LSPR) of the well or post.
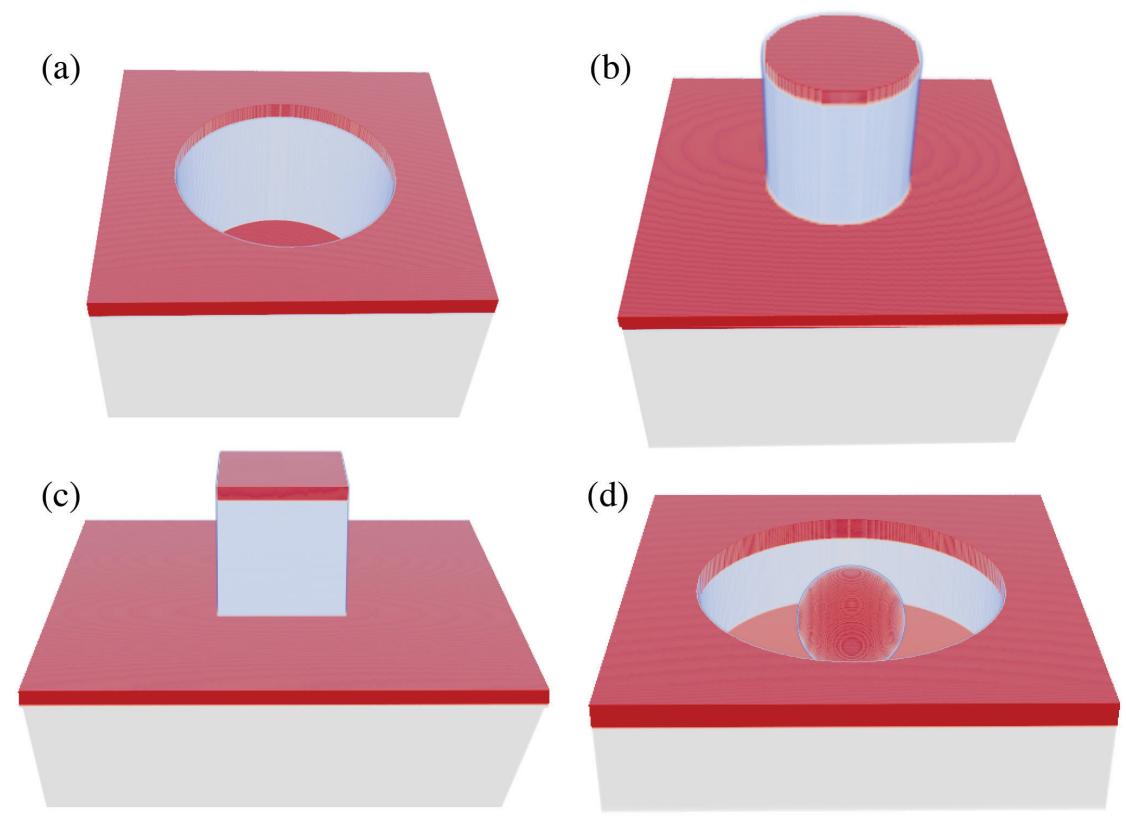

Figure 1. Unit cell geometries for (a) nanowells, (b) cylindrical nanoposts, (c) square nanoposts, and (d) particle imbedded nanowells. Plasmonic crystals result from the use of periodic boundary conditions.

The present work aims to re-examine these plasmonic crystal studies as a means to compare the predictive abilities of two related FDTD approaches in estimating relative SERS responses. The FDTD method is a numerical approach in which electric and magnetic field components are propagated on a set of discrete grid points, each described by a dielectric constant consistent with the material in the structure being modeled at that point [15]. FDTD is a powerful tool for modeling the interactions of light with arbitrarily shaped, complex geometric structures and has been used in a wide variety of surface plasmon applications, including sensing, photovoltaics, imaging, optical trapping, metamaterials and optical transparency, and, of course, SERS [16-24]. With FDTD electric field intensities calculated at both excitation and scattered wavelengths, $\lambda_{\text {exc }}$ and $\lambda_{\text {scat }}$, respectively, the SERS response can be calculated as 


$$
G_{\text {SERS }}^{4}=\frac{1}{N} \sum_{x_{i}} \sum_{y_{j}} \sum_{z_{k}} g^{2}\left(x_{i}, y_{j}, z_{k}, \lambda_{\text {exc }}\right) \times g^{2}\left(x_{i}, y_{j}, z_{k}, \lambda_{\text {scat }}\right),
$$

where $x_{i}, y_{j}$, and $z_{k}$ are FDTD grid points and $N$ is the number of grid points in the volume of air near the surface of a metallic nanostructure $[23,25,26]$. Equation (1) requires the computation of fields at $\lambda_{\text {exc }}$ and $\lambda_{\text {scat }}$. For large three-dimensional grids, due either to a large spatial extent or a fine grid resolution, or for long simulation times, it is possible to reduce computational cost by approximating the SERS response as a function of a single wavelength, typically $\lambda_{\text {exc }}$ or $\lambda_{\text {mid }}$, the midpoint between the excitation and scattered wavelenghts $[13,14,27,28]$. The use of $\lambda_{\text {exc }}$ is suitable if the Stokes shift is small compared with $\lambda_{\text {exc }}$. The use of $\lambda_{\text {mid }}$ is suitable if the surface plasmon resonance is sufficiently broad over the range of excitation and scattered wavelenghts. Early studies of colloidal gold nanospheres indeed showed that particles with an LSPR parked between the excitation and scattered wavelengths were able to effectively enhance both $\lambda_{\text {exc }}$ and $\lambda_{\text {scat }}$ to produce the highest SERS response [29]. The SERS response based on this approximation using $\lambda_{\text {mid }}$, referred to hereafter as $G_{\text {mid }}^{4}$, is given by

$$
G_{\text {mid }}^{4}=\frac{1}{N} \sum_{x_{i}} \sum_{y_{j}} \sum_{z_{k}} g^{4}\left(x_{i}, y_{j}, z_{k}, \lambda_{\text {mid }}\right) .
$$

The aim of this study, then, was to compare Equations (1) and (2) in estimating relative SERS responses for NW and NP plasmonic crystals, which are able to support multiple LSPRs with complicated coupling.

In Section 2.1, we show that the former approach is necessary to get more quantitative relative SERS responses. With this in mind, we then set out to design and optimize SERS substrates in silico without fabrication costs. In Refs. $[13,14]$, the dimensions studied experimentally do not allow one to distinguish between the effect of diameter and the effect of periodicity on the SERS response, both of which can affect a SP resonance. In Section 2.2, we optimize of NW and NP geometries with respect to well depth/post height, diameter, and periodicity using Equation (1). In Section 2.3, we present two studies looking at novel plasmonic crystals based on variations of NW and NP geometries. Differences include nanoposts with square cross-sections (Figure 1c) and nanowells with imbedded nanoparticles (Figure 1d).

\section{Results}

\subsection{Comparison of FDTD Simulated SERS Responses for Nanowell and Nanopost Plasmonic Crystals}

Time-averaged electric field intensity enhancements were calculated for $\lambda=785,821$, and $857 \mathrm{~nm}$ using the finite-difference time-domain (FDTD) method for a series of nanowell (NW, Figure 1a) and cylindrical nanopost (NP, Figure 1b) geometries. For the NW geometries, an SU-8 support with refractive index $n=1.59$ was used with a relief depth $R D=360 \mathrm{~nm}$ and gold metal thickness $M T=40 \mathrm{~nm}$ [13]. For the NP geometries, an NOA support with refractive index $n=1.56$ was used with a relief depth $R D=200 \mathrm{~nm}$ and gold metal thickness $M T=24 \mathrm{~nm}$ [14]. All parameters were consistent with fabricated arrays. Periodic boundary conditions were implemented in the $x$ - and $y$-directions to simulate the array. Periodicities and well/post diameters considered are listed in Table 1.

SERS responses $G_{\text {SERS }}^{4}$ and $G_{\text {mid }}^{4}$ were calculated using Equations (1) and (2), respectively. Figure 2a contains a plot for the NW geometries comparing the FDTD simulated SERS responses (open symbols) with experimental measurements (filled circles) reported in Ref. [13]. (Solid and dashed/dotted lines correspond to spline interpolation between data points and are meant for ease of visualization only.) 
Table 1. Periodicities and diameters for experimental plasmonic crystals.

\begin{tabular}{cc}
\hline Diameter (nm) & Periodicity (nm) \\
\hline 174 & 490 \\
224 & 584 \\
256 & 658 \\
456 & 730 \\
500 & 800 \\
514 & 760 \\
616 & 1000 \\
685 & 1100 \\
\hline
\end{tabular}
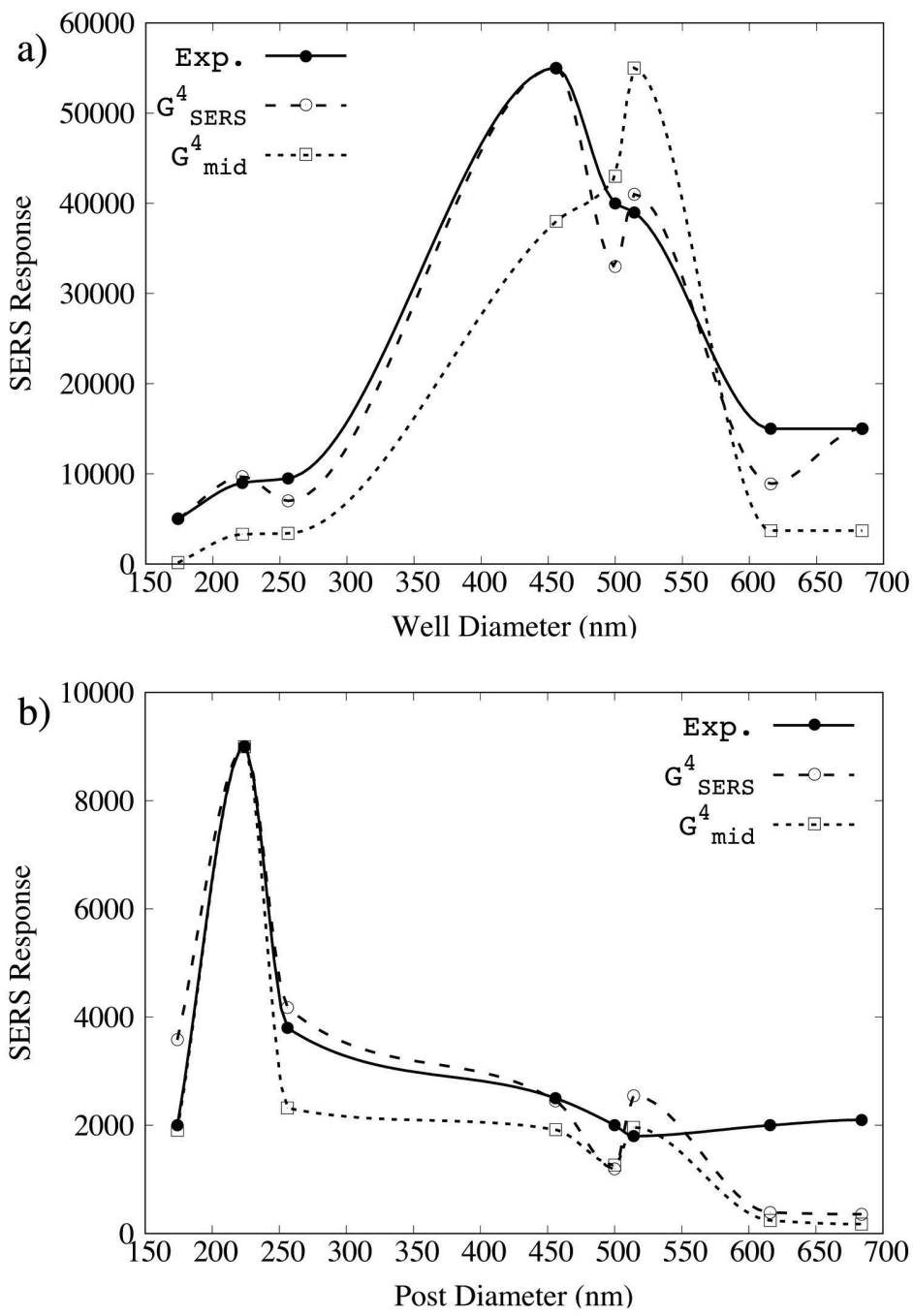

Figure 2. Comparison of experimental SERS response (exp) and calculated SERS responses ( $G_{\text {SERS }}^{4}$ and $G_{\text {mid }}^{4}$ ) for the (a) NW and (b) NP geometries. Experimental SERS response for the NW and NP geometries are taken from Refs. [13,14], respectively. Experimental values are plotted with filled circles. SERS responses using Equation (1) are plotted with open circles. SERS responses calculated using Equation (2) are plotted with open squares. Solid and dashed/dotted lines correspond to spline interpolation between data points and are meant for ease of visualization only. Calculated SERS responses are scaled such that maximum values equal the experimental maximum.

Experimental Raman intensity measurements reveal a maximum at a diameter $D=456 \mathrm{~nm}$ $(P=730 \mathrm{~nm})$ at 55,000 counts. Experimental SERS spectra were collected for a $15 \mathrm{mM}$ solution of 
benzene thiol using a dispersive Raman microscope. The units are not directly comparable to $G_{\text {SERS }}^{4}$ and $G_{\text {mid }}^{4}$, which are based on unitless electric field intensity enhancements. The FDTD SERS responses, therefore, are scaled such that the maximum experimental and theoretical values coincide. It is clear from Figure 2a that $G_{\text {SERS }}^{4}$, using Equation (1), reproduces the experimental SERS responses better than $G_{\text {mid }}^{4}$, using Equation (2). Differences between the $G_{\text {SERS }}^{4}$ and $G_{\text {mid }}^{4}$ results can be understood in terms of the exictation of different LSPRs for $D=456 \mathrm{~nm}$ and $D=514 \mathrm{~nm}$ at $785 \mathrm{~nm}, 821 \mathrm{~nm}$, and $857 \mathrm{~nm}$, and predicted SERS response based on $\lambda_{\text {mid }}$ alone can be misleading.

In a similar comparison, Figure $2 b$ contains a plot for the NP geometries comparing the FDTD simulated SERS responses (dashed lines with open symbols) with experimental measurements (solid line with filled circles) reported in Ref. [14]. Experimental Raman intensity measurements reveal a maximum at a diameter $D=224 \mathrm{~nm}(P=584 \mathrm{~nm})$ at 9000 counts, and the FDTD SERS responses are again scaled such that the maximum experimental and theoretical values coincide. (It should also be noted that the units for the experimental SERS response for the NWs and the NPs are not exactly the same, and it turns out that NP arrays produce higher SERS responses than similar NW geometries [14].) While the agreement between $G_{\text {SERS }}^{4}$ and $G_{\text {mid }}^{4}$ are more similar in terms of predicted SERS responses, the $G_{\text {SERS }}^{4}$ values are slightly better in a least squares sense. The better agreement in the NP case can be understood as less coupling possible between the disc and the metal film, in contrast to the NW case where the void, film, and lower disc can couple, as evidenced by the differences in the transmission spectra seen in Figure 3.

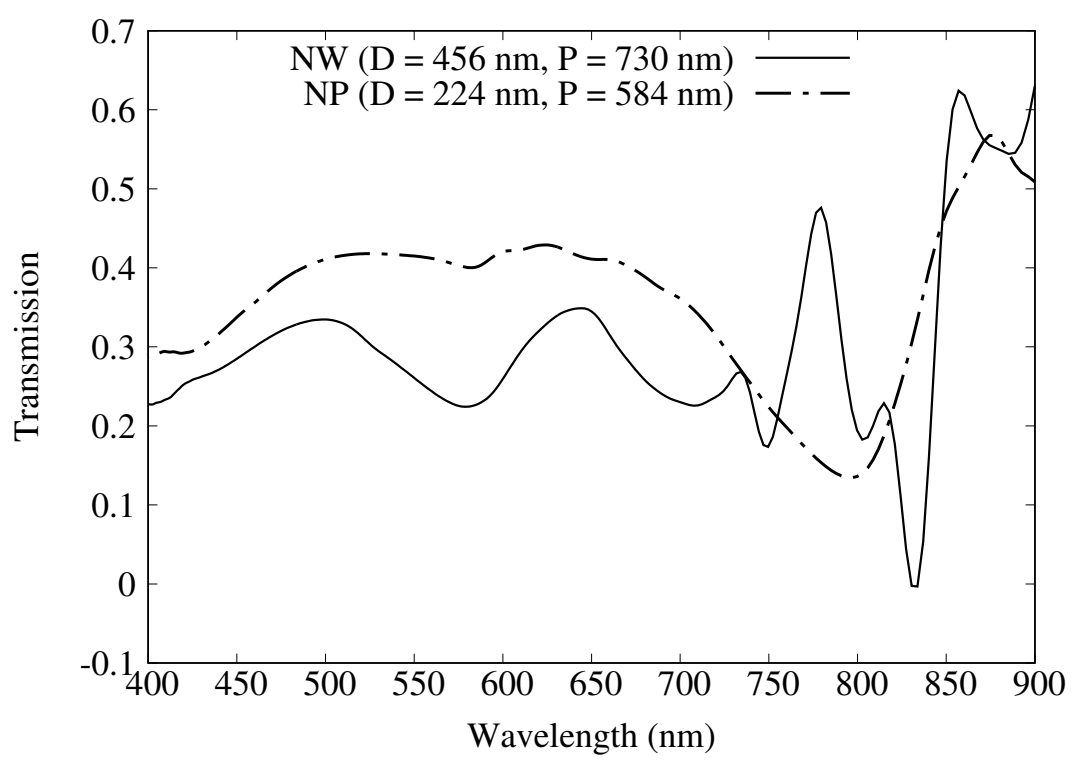

Figure 3. Plot of transmission for nanowell (solid) and nanopost (dashed).

\subsection{Optimization of FDTD Simulated SERS Responses for Nanowell and Nanopost Plasmonic Crystals}

The better quantitative agreement between $G_{\text {SERS }}^{4}$ and $G_{\text {exp }}^{4}$ allows us to optimize relative SERS responses for the NW and NP geometries by scanning geometry parameter space, allowing the relief depth $(R D)$, diameter $(D)$, periodicity $(P)$, and metal thickness $(M T)$ to vary and calculating the resulting relative SERS response. We define the NW geometry with $D=456 \mathrm{~nm}, P=730 \mathrm{~nm}, R D=360 \mathrm{~nm}$, and $M T=40 \mathrm{~nm}$ as the NW "control". We also define an optimization factor

$$
\text { O.F. }=\frac{G_{\text {SERS }}^{4}}{G_{\text {control }}^{4}}
$$

such that any O.F. $>1$ results in an enhancement of the expected SERS response. Figure 4 contains a plot of optimization factors as the nanowell $R D, D, P$, and $M T$ were varied sequentially. 

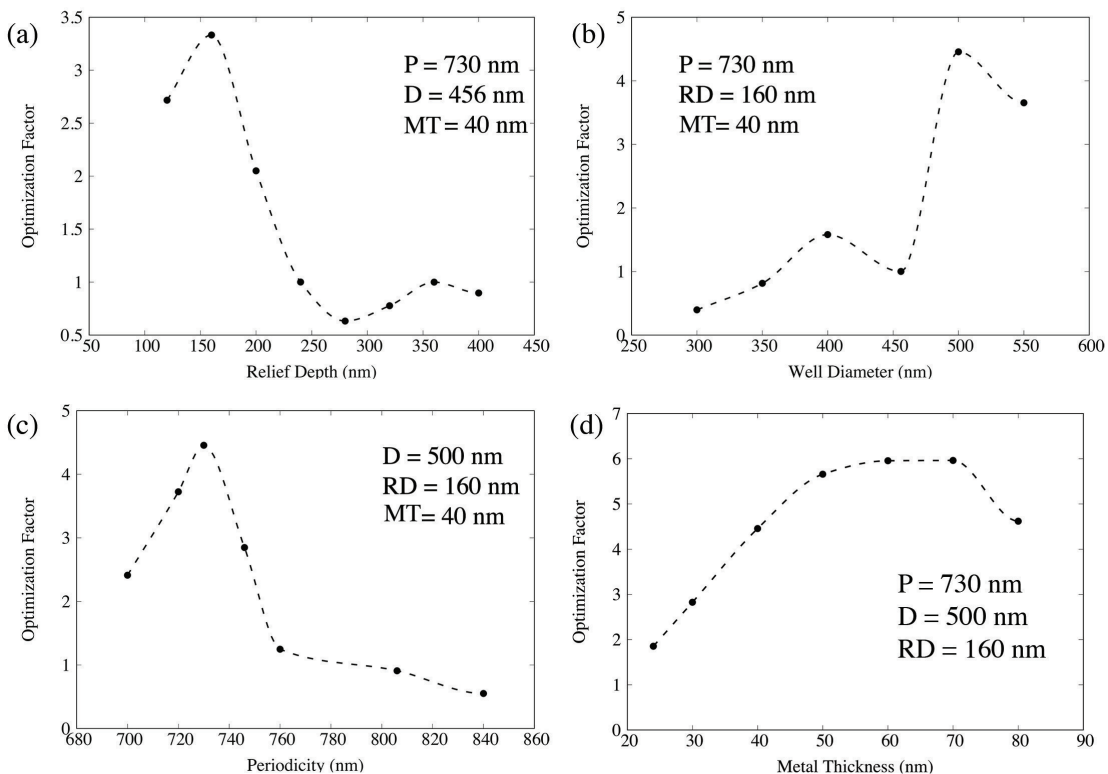

Figure 4. Plot of optimization factors (O.F. $=G_{\text {SERS }}^{4} / G_{\text {control }}^{4}$ ) as nanowell (a) relief depth, (b) diameter, (c) periodicity, and (d) metal thickness were sequentially varied. The final optimization factor is 6.0. Dashed lines correspond to spline interpolation between data points and are meant for ease of visualization only.

The final optimization factor is 6.0, indicating a nanowell plasmonic crystal with $P=730 \mathrm{~nm}$, $D=500 \mathrm{~nm}, R D=160 \mathrm{~nm}$, and $M T=70 \mathrm{~nm}$ would give rise to a $6 \times$ increased SERS signal over the experimentally optimum structure. Figure 5 shows a comparison of electric field enhancements at $\lambda=785 \mathrm{~nm}$ and $\lambda=857 \mathrm{~nm}$ for the control (Figure 5a) and optimized (Figure 5b) arrays, respectively. Increased field intensities in the well region lead to a higher SERS response.

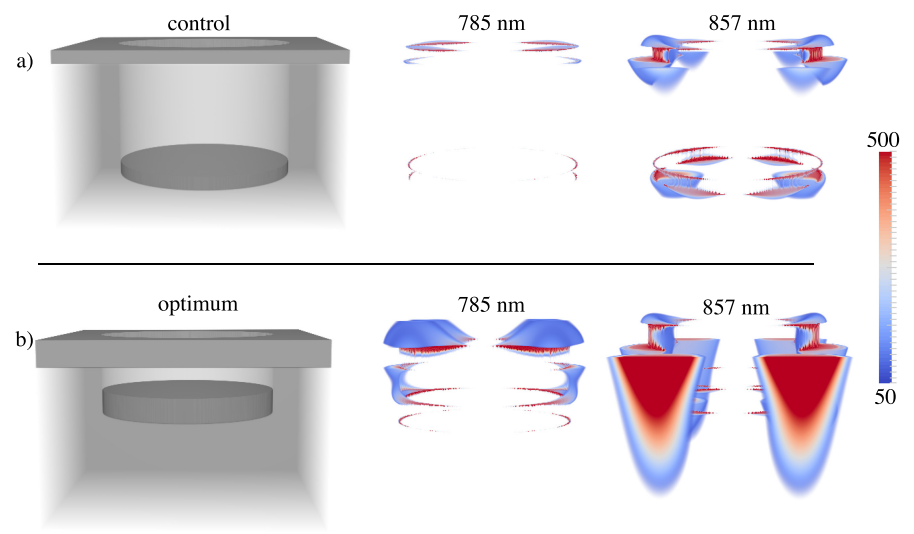

Figure 5. Plot of dielectric constant and time-averaged electric field intensity enhancements $\left(g^{2}=|E|^{2} /\left|E_{0}\right|^{2}\right)$ at $\lambda=785 \mathrm{~nm}$ and $\lambda=857 \mathrm{~nm}$ for (a) unit cell of control NW geometry ( $D=456 \mathrm{~nm}, P=730 \mathrm{~nm}$, $R D=360 \mathrm{~nm}$, and $M T=40 \mathrm{~nm})$ and $(\mathbf{b})$ unit cell of optimized NW geometry $(D=500 \mathrm{~nm}, P=730 \mathrm{~nm}$, $R D=160 \mathrm{~nm}$, and $M T=70 \mathrm{~nm}$ ). Only enhancements in the 50-500 range are plotted.

In a similar approach, the "control NP" geometry was defined as $D=224 \mathrm{~nm}, P=584 \mathrm{~nm}$, $R D=200 \mathrm{~nm}$, and $M T=24 \mathrm{~nm}$. By varying $R D, D, P$, and $M T$, an optimized NP geometry was determined to have $D=200 \mathrm{~nm}, P=720 \mathrm{~nm}, R D=210 \mathrm{~nm}$, and $M T=24 \mathrm{~nm}$ with an O.F. $=2.0$. Given the similarity in the optimized and control post diameters and relief depths, the increase came predominantly through the increase in the periodicity (Figure 6). 


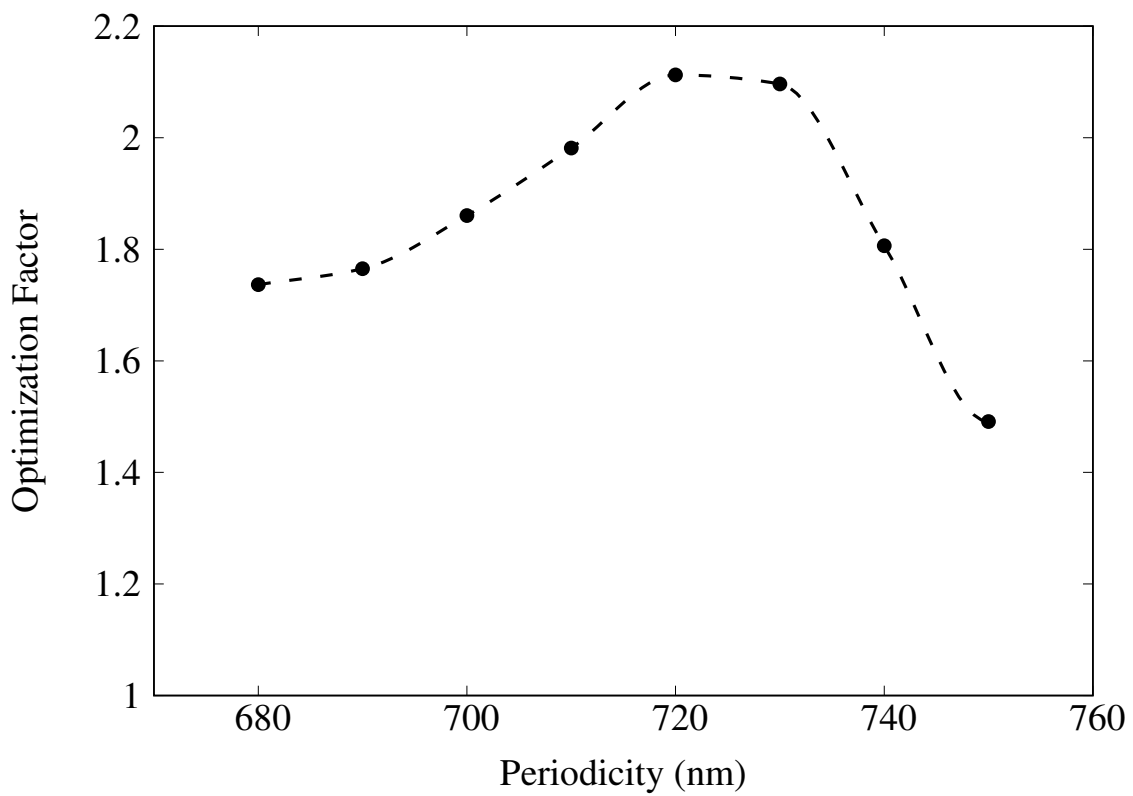

Figure 6. Plot of optimization factor, O.F., as a function of periodicity for cylindrical NP geometry with $R D=210 \mathrm{~nm}, D=200 \mathrm{~nm}$, and $M T=24 \mathrm{~nm}$ in the range $680-750 \mathrm{~nm}$. Dashed lines correspond to spline interpolation between data points and are meant for ease of visualization only.

\subsection{Optimization of FDTD Simulated SERS Responses for Novel Plasmonic Crystals}

In this section, we describe calculations aimed at increasing relative SERS responses even further by considering new geometries that should support either increased field intensities and/or additional coupling at $785 \mathrm{~nm}$ and $857 \mathrm{~nm}$. To this end, we considered a square nanopost (Figure 1c) array and a nanowell array with imbedded nanoparticles (Figure 1d). The motivation for considering the square post was to excite local surface plasmons at the sharper corners, and such an array could easily be fabricated using the same soft-nanolithography techniques used to construct the cylindrical nanoposts considered above. The square NPs were positioned such that edges of length $D$ were oriented along the $x$ - and $y$-directions. In order to couple into surface plasmons at the corners, we used incident light polarized at $45^{\circ}$. We considered the following range of values: $D=130-500 \mathrm{~nm}, R D=150-260 \mathrm{~nm}$, $P=500-800 \mathrm{~nm}$, and $M T=6-20 \mathrm{~nm}$. An optimum square NP was found to have an optimization factor of $O . F .=6.3$ with $D=150 \mathrm{~nm}, R D=190 \mathrm{~nm}, P=730 \mathrm{~nm}$, and $M T=24 \mathrm{~nm}$. Figure 7 depicts the structure and fields at $785 \mathrm{~nm}$. (Field distributions at $857 \mathrm{~nm}$ were similar to $785 \mathrm{~nm}$ and are not presented here.)

For the plasmonic crystals considered thus far, optimum SERS responses are associated with the coupling of local surface plasmons of the film and either of the void (NW) or post (NP), as indicated by periodicity and shape affect on the electric field intensities. We also considered imbedding spherical gold nanoparticles in NW geometries. The aim of this study was to increase electric field intensities by allowing coupling between the particle and the perforated film as well as between the particle and the well disc. Beginning with the optimum NW geometry from Section 2.2, we calculated $G_{\text {SERS }}^{4}$ for a range of particle diameters, from $80 \mathrm{~nm}$ to $300 \mathrm{~nm}$. Optimization factors are plotted in Figure 8 . The control NW geometry is the same as used in Section 2.2, namely $D=456 \mathrm{~nm}, P=730 \mathrm{~nm}, R D=360 \mathrm{~nm}$, and $M T=40 \mathrm{~nm}$. 


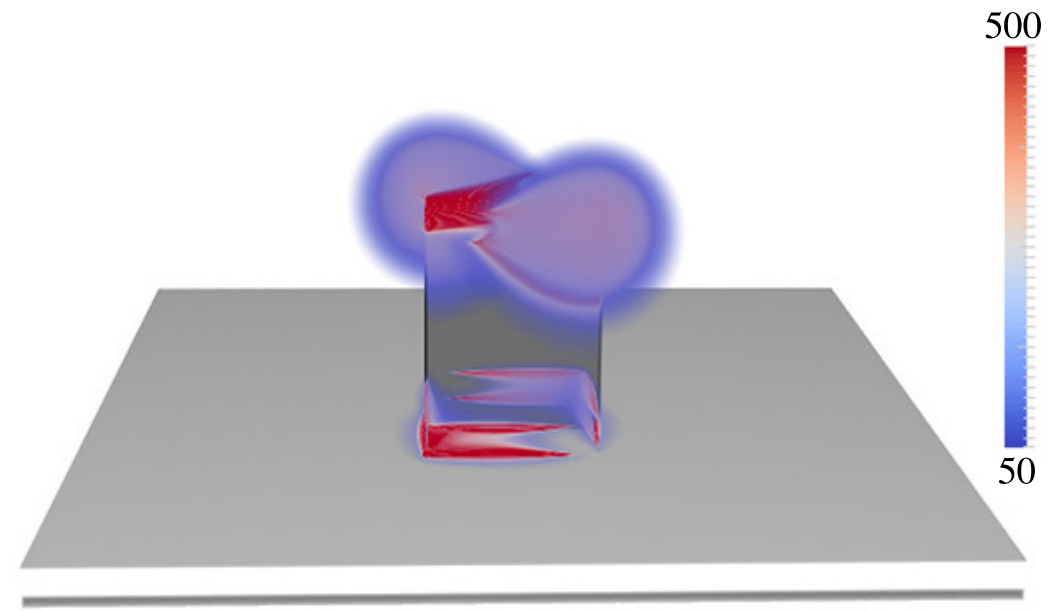

Figure 7. Plot of electric field intensity enhancement $\left(g^{2}=|E|^{2} /\left|E_{0}\right|^{2}\right)$ at $\lambda=785 \mathrm{~nm}$ for optimized square NP. The optimal parameters are $D=150 \mathrm{~nm}, R D=190 \mathrm{~nm}, P=730 \mathrm{~nm}$, and $M T=24 \mathrm{~nm}$ with an O.F. $=6.3$. Only enhancements in the 50-500 range are plotted.

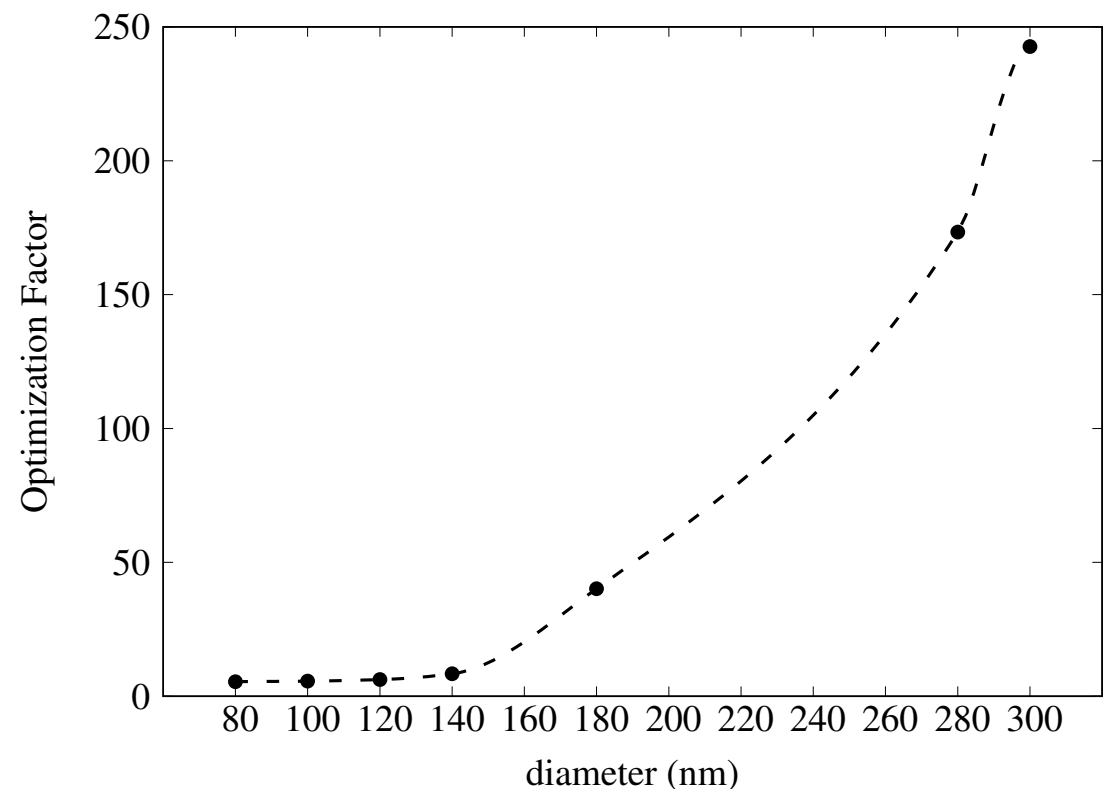

Figure 8. Plot of optimization factors as a function of particle diameter for spherical gold nanoparticles imbedded in an NW plasmonic crystal. Dashed lines correspond to spline interpolation between data points and are meant for ease of visualization only.

It is not surprising that $G_{\text {SERS }}^{4}$ (and hence O.F.) increases with increasing particle diameter. While isolated solid gold nanospheres do not absorb strongly in the near infrared, scattering increases for increasing particle size. Figure 9 shows the extinction efficiency (extinction cross section per geometrical cross section) for a $300 \mathrm{~nm}$ gold sphere. A broad peak is seen above $700 \mathrm{~nm}$. The increased scattering as well as the coupling to the nanowell gives rise to increasingly large enhancements.

An initial study varying relief-depth, diameter, periodicity, and metal thickness sequentially revealed highest $G_{\text {SERS }}^{4}$ values for periodicities near $730 \mathrm{~nm}$ and well diameters near $600 \mathrm{~nm}$. We then calculated $G_{\mathrm{SERS}}^{4}$ for all combinations of $P=700,730$, and $760 \mathrm{~nm}, D=550,600$, and $650 \mathrm{~nm}, R D=100$, 140, and $180 \mathrm{~nm}$, and $M T=40$ and $70 \mathrm{~nm}$ (see Table 2). 


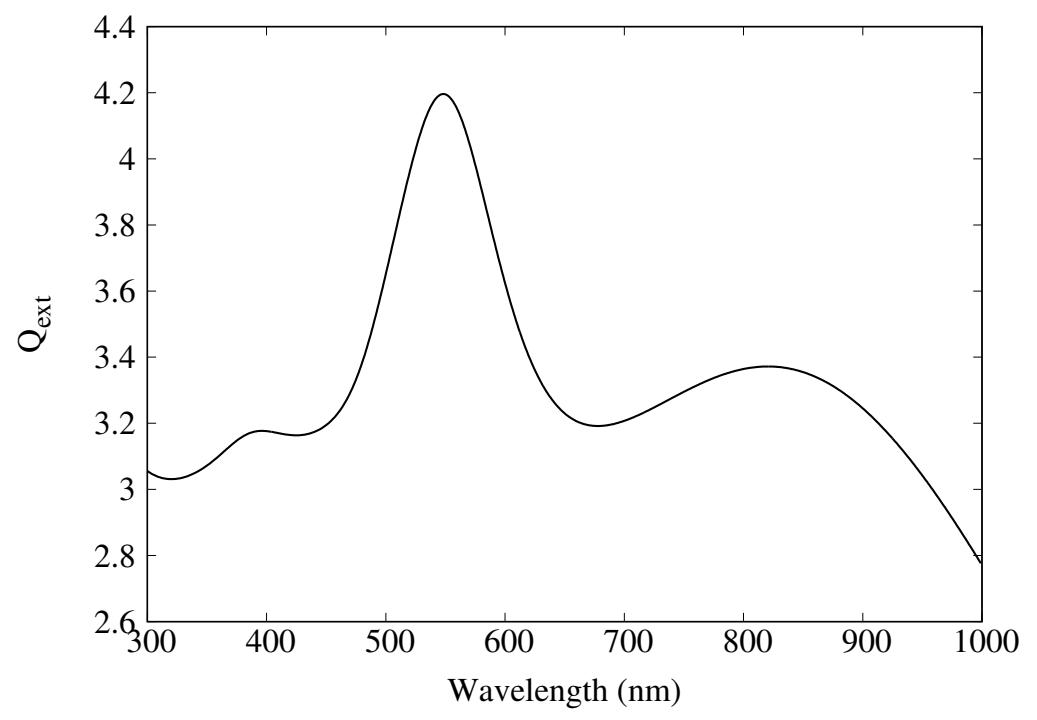

Figure 9. Extinction efficiency, $Q_{\text {ext }}$, for a $300 \mathrm{~nm}$ diameter spherical gold nanoparticle.

Table 2. Optimization factors for nanowell geometries imbedded with $300 \mathrm{~nm}$ spherical gold nanoparticles.

\begin{tabular}{ccccc}
\hline $\mathbf{P}(\mathbf{n m})$ & $\mathbf{D}(\mathbf{n m})$ & $\mathbf{R D}(\mathbf{n m})$ & $\begin{array}{c}\text { O.F. } \\
(\boldsymbol{M} \boldsymbol{T}=\mathbf{4 0} \mathbf{n m})\end{array}$ & $\begin{array}{c}\text { O.F. } \\
(\boldsymbol{M} \boldsymbol{T}=\mathbf{7 0} \mathbf{n m})\end{array}$ \\
\hline 700 & 550 & 100 & 1163 & 917 \\
700 & 550 & 140 & 401 & 366 \\
700 & 550 & 180 & 320 & 213 \\
700 & 600 & 100 & 1815 & 1171 \\
700 & 600 & 140 & 619 & 751 \\
700 & 600 & 180 & 452 & 460 \\
700 & 650 & 100 & 2358 & 1405 \\
700 & 650 & 140 & 887 & 966 \\
700 & 650 & 180 & 627 & 616 \\
730 & 550 & 100 & 870 & 600 \\
730 & 550 & 140 & 223 & 307 \\
730 & 550 & 180 & 147 & 181 \\
730 & 600 & 100 & 1538 & 852 \\
730 & 600 & 140 & 381 & 411 \\
730 & 600 & 180 & 184 & 211 \\
730 & 650 & 100 & 1920 & 1156 \\
730 & 650 & 140 & 889 & 897 \\
730 & 650 & 180 & 647 & 656 \\
760 & 550 & 100 & 463 & 561 \\
760 & 550 & 140 & 52 & 143 \\
760 & 550 & 180 & 16 & 47 \\
760 & 600 & 100 & 837 & 248 \\
760 & 600 & 140 & 201 & 195 \\
760 & 600 & 180 & 164 & 437 \\
760 & 650 & 100 & 1050 & 351 \\
760 & 650 & 140 & 347 & \\
760 & 650 & 180 & 292 & \\
\hline
\end{tabular}

Calculated O.F. values ranged from one to three orders of magnitude larger than the NW alone, with $M T=40$ predominantly giving rise to larger O.F. values overall. Figure 10 contains a plot of the time-averaged electric field intensity at $785 \mathrm{~nm}$ and $857 \mathrm{~nm}$ for the optimal geometry: $D=650 \mathrm{~nm}$, $R D=100 \mathrm{~nm}, P=700 \mathrm{~nm}$, and $M T=40 \mathrm{~nm}$ with an O.F. $=2400$. It is clear that the coupling of the nanoparticle to the well disc is the leading contribution to the increased $G_{\mathrm{SERS}}^{4}$. In order to assess the 
extent of coupling to the disc verses the film, we calculated $G_{\text {SERS }}^{4}$ and associated O.F. when the metal film was removed (replaced with SU-8). The resulting O.F. $=1010$ shows that there is a factor of two contribution of enhancement due to the coupling of the film to the particle.
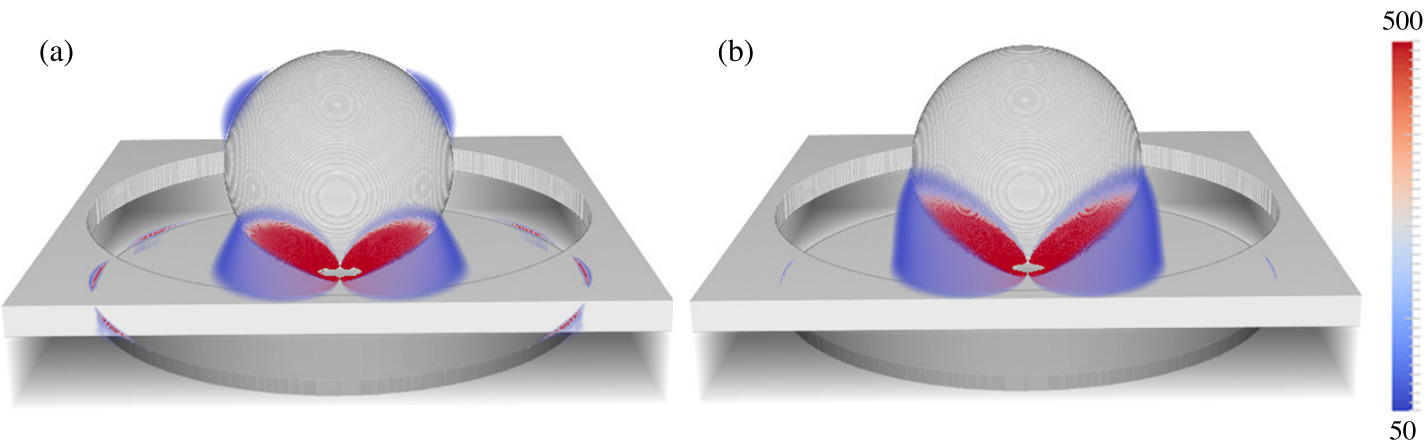

Figure 10. Plot of electric field intensity at (a) $\lambda=785 \mathrm{~nm}$ and (b) $\lambda=857 \mathrm{~nm}$ for optimized NW with imbedded $300 \mathrm{~nm}$ diameter nanoparticles. The optimal parameters are $D=650 \mathrm{~nm}, R D=100 \mathrm{~nm}$, $P=700 \mathrm{~nm}$, and $M T=40 \mathrm{~nm}$ with an O.F. $=2400$.

\section{Discussion}

In this work, we set out to use the FDTD method to calculate electric field intensity enhancements at $\lambda_{\text {exc }}, \lambda_{\text {mid }}$, and $\lambda_{\text {scat }}$ for a series of plasmonic crystals to compare $G_{\text {SERS }}^{4}$ and $G_{\text {mid }}^{4}$, as defined in Equations (1) and (2), in their ability to reproduced experimental measurements. By using the word relative, we mean that maximum $G_{\text {SERS }}^{4}$ and $G_{\text {mid }}^{4}$ are scaled to match maximum experimental values. The scaling translates the unitless electric field intensity enhancement, $g^{2}$, into units consistent with experimental SERS measurements, and as structure parameters are varied, increases or decreases in the SERS response can be predicted. For both geometries, $G_{\text {SERS }}^{4}$ understandably gives rise to better quantitative agreement with experimental SERS measurements. For the NP geometry, $G_{\text {mid }}^{4}$ is a good approximation to $G_{\text {SERS }}^{4}$ due to the broad spectral feature in the $\lambda_{\text {exc }}-\lambda_{\text {scat }}$ range (Figure 3). However, for the NW geometry which supports more complex coupling between multiple SP resonances in this range, $G_{\text {mid }}^{4}$ failed to produce even qualitative agreement for some arrays. These results are not surprising; after all, $G_{\text {mid }}^{4}$ is an approximation, and when underlying assumptions break down, the accuracy of an approximation also suffers. However, they do suggest caution in using $G_{\text {mid }}^{4}$ for predictive applications involving complex nanostructures for SERS. It is important to note that the better quantitative agreement of $G_{\text {SERS }}^{4}$ comes at a computational cost given simulations at both excitation and scattering wavelengths must be considered.

It is also important to note that alternate strategies could be used to estimate SERS responses. While Equations (1) and (2) represent an average over all grid points corresponding to air in the vicinity of the metal surface, one could envision using only maximum values of $G_{\text {mid }}^{4}$ or $G_{\text {SERS }}^{4}$ or averaging over only values above some threshold. While we do not present the results here, we also performed estimations of SERS responses based on these two alternate approaches and found that neither case reproduced relative experimental as well as an average over all grid points in a volume of air near each NW or NP.

We also set out to design and optimize plasmonic crystals in silico by varying structure parameters, such as relief depth $(R D)$, well/post diameter $(D)$, periodicity $(P)$, metal thickness $(M T)$, and post shape to maximize excitation and/or coupling of local surface plasmons. While only modest improvements were seen for the cylindrical nanopost, we saw a $6 \times$ increase in the NW geometry and the square NP geometry over their respective control structures. For the cylindrical NW and NP geometries and for the square NP geometry, we used a "sequential" optimization scheme, which involved varying only one parameter at a time, updating that parameter with the new optimum value before proceeding to the next parameter. This sequential approach enabled us to look at a finer grid of values for each 
parameter, but it is important to note that better geometries might have been excluded, as changing one parameter can affect optimum values of another previously determined. One improved approach would be to perform a more self consistent approach where we cycle through sequentially until convergence of parameters. Another approach would be to use the sequential method to identify potential optimum values for each parameter and then consider all combinations of that courser set of parameters (as was done in the case of imbedded nanoparticles in this Section 2.3 for the NW with imbedded nanoparticles.) It is likely an order of magnitude improvement over the control would be seen for the the NW and square NP geometries. In regards to fabrication, it is important to note that the novel square NP geometry presented herein should both be easily be fabricated using soft-nanolithograpy [9].

By far the most dramatic increases in predicted SERS responses arise from the addition of $300 \mathrm{~nm}$ gold nanoparticles to the NW geometry, with optimization factors ranging from $10 \times$ to $2000 \times$, with an average of $600 \times$ increase in the predicted SERS response. In regards to fabrication, particles up to $300 \mathrm{~nm}$ can be synthesized with a uniform spherical shape and narrow size distribution using a combination of chemical reduction and annealing [30]. The challenge, though, is an even distribution of particles within the wells and not on the top metal film. We believe it is possible for the following reasons. As seen in Table 2, the optimum NW geometry with imbedded particles consisted of a $700 \mathrm{~nm}$ periodicity and a $650 \mathrm{~nm}$ well. The wells, then, correspond to almost $70 \%$ of the total surface area. By controlling the concentration of $300 \mathrm{~nm}$ particles, configurations with a single particle in a well should be statistically favored.

Finally, while the structures herein were optimized for the benzene thiol band at $1073 \mathrm{~cm}^{-1}$, it is important to note that plasmonic crystals can be designed and optimized using FDTD for any particular fingerprint Raman band for any analyte of interest. The significance of this work is in showing the potential of using FDTD to design and optimize novel plasmonic crystals in lieu of a costly trial-and-error fabrication approach.

\section{Materials and Methods}

To model the interactions of light with the nanowell/nanopost plasmonic crystals, we used Meep [31], a full three-dimensional finite-difference time-domain (FDTD) solver for Maxwell's curl equations. In the FDTD method, electric and magnetic fields are represented on a discrete, staggered grid and are propagated in time in using a leap-frog algorithm [15]. The material composition at each grid point is specified by a dielectric constant consistent with the spatial layout of each plasmonic crystal. Dielectric supports were modeled with a constant (non-dispersive) refractive index in line with photopolymers used in fabricated arrays: SU-8 $(n=1.59)$ [13] for NW geometries and NOA $(n=1.56)$ [14] for NP geometries. Gold was modeled using a dispersive Drude plus two Lorentzian fit of Johnson and Christy's dielectric data [32] over 300-1000 nm:

$$
\epsilon_{\mathrm{Au}}=\epsilon_{\infty}-\frac{\omega_{D}^{2}}{\omega+i \omega \gamma_{D}}-\sum_{m=1}^{2} \frac{\sigma_{m} \omega_{m}^{2}}{\omega^{2}-\omega_{m}^{2}+i \omega \gamma_{m}}
$$

with $\epsilon_{\infty}=4.05665, \omega_{D}=8.75866 \mathrm{eV}, \gamma_{D}=0.04062 \mathrm{eV}, \omega_{1}=2.92893 \mathrm{eV}, \gamma_{1}=0.83423 \mathrm{eV}, \sigma_{1}=1.09960$, $\omega_{2}=4.05286 \mathrm{eV}, \gamma_{2}=1.74845 \mathrm{eV}$, and $\sigma_{3}=2.2195$. Periodic boundary conditions in the $x$ - and $y$-directions were used to simulate square arrays. Meep's perfectly matched layers (PML) were used as absorbing boundary conditions at the top and bottom of the computational cell to prevent spurious reflections [33]. The computational box size in the $z$-direction was $1500 \mathrm{~nm}$ with a grid spacing of $2 \mathrm{~nm}$ and $60 \mathrm{~nm}$ of PML in both directions. Light was propagated in the $z$-direction from air from a line source at the air/PML interface. With $\theta_{\text {src }}$ defined such that $\theta_{\text {src }}=0^{\circ}$ corresponds to an $x$-polarized light source and $\theta_{\mathrm{src}}=90^{\circ}$ corresponds to a $y$-polarized light source, $\theta_{\text {src }}=0^{\circ}$ was used for all NW and cylindrical NP geometries, and $\theta_{\mathrm{src}}=45^{\circ}$ was used for the square NP geometries. Electric fields for $\lambda=785,821$, and $857 \mathrm{~nm}$ were propagated for 100 fs to ensure complete coupling into surface 
plasmons. Electric field intensities, with and without plasmonic crystals present, were averaged over the last two periods $(c / \lambda)$ to get the time-averaged electric field intensity enhancement, $g^{2}=|E|^{2} /\left|E_{0}\right|^{2}$, at each wavelength. We calculated $G_{\text {SERS }}^{4}$ and $G_{\text {mid }}^{4}$ (Equations (1) and (2)) by averaging over $g^{2}$ only for points in air within $100 \mathrm{~nm}$ above the NW or NP. Points in the gold film or dielectric support were excluded from the average since they would not be accessible to a target molecule, and the $100 \mathrm{~nm}$ range from the NW or NP allowed for electric fields corresponding to surface plasmons to sufficiently decay. Zero-order transmission (T) spectra were calculated by taking the ratio of the transmitted power to the incident power integrated over a plane within the dielectric support. The extinction cross section for the $300 \mathrm{~nm}$ gold sphere was calculated using Mie theory [5,34]. Plots of electric field intensities were generated using ParaView $[35,36]$.

Author Contributions: A.B. and J.M. conceived and designed the computational experiments. J.M. and A.B. performed the nanowell and nanopost simulations, respectively. J.M. wrote the paper.

Acknowledgments: This work used resources of the National Energy Research Scientific Computing Center, which is supported by the Office of Science of the U.S. Department of Energy under Contract No. DE-AC02-05CH11231.

Conflicts of Interest: The authors declare no conflict of interest.

\section{References}

1. Fleischmann, M.; Hendra, P.J.; McQuillan, A.J. Raman spectra of pyridine adsorbed at a silver electrode. Chem. Phys. Lett. 1974, 26, 163-166. [CrossRef]

2. Albrecht, M.G.; Creighton, J.A. Anomalously intense Raman spectra of pyridine at a silver electrode. J. Am. Chem. Soc. 1977, 99, 5215-5217. [CrossRef]

3. Van Duyne, R.P.; Hulteen, J.C.; Treichel, D.A. Atomic force microscopy and surface-enhanced Raman spectroscopy. I. Silver island films and silver film over polymer nanosphere surfaces supported on glass. J. Chem. Phys. 1993, 99, 2101. [CrossRef]

4. Raether, H. Surface Plasmons on Smooth and Rough Surfaces and on Gratings; Spring-Verlag: Berlin/Heidelberg, Germany, 1988.

5. Bohren, C.F.; Huffman, D.R. Absorption and Scattering of Light by Small Particles; Wiley: New York, NY, USA, 1983.

6. Kelly, K.L.; Coronado, E.; Zhao, L.L.; Schatz, G.C. The Optical Properties of Metal Nanoparticles: The Influence of Size, Shape, and Dielectric Environment. J. Phys. Chem. B 2003, 107, 668-677. [CrossRef]

7. Eustis, S.; El-Sayed, M.A. Why gold nanoparticles are more precious than pretty gold: Noble metal surface plasmon resonance and its enhancement of the radiative and nonradiative properties of nanocrystals of different shapes. Chem. Soc. Rev. 2006, 305, 209-217. [CrossRef] [PubMed]

8. Yang, W.H.; Hulteen, J.; Schatz, G.C.; Van Duyne, R. A surface-enhanced hyper-Raman and surface-enhanced Raman scattering study of trans-1,2-bis(4-pyridyl)ethylene adsorbed onto silver film over nanosphere electrodes. Vibrational assignments: Experiment and theory. J. Chem. Phys. 1996, 104, 4313-4323. [CrossRef]

9. Stewart, M.E.; Mack, N.H.; Malyarchuk, V.; Soares, J.A.N.T.; Lee, T.W.; Gray, S.K.; Nuzzo, R.G.; Rogers, J.A. Quantitative multispectral biosensing and 1D imaging using quasi-3D plasmonic crystals. Proc. Natl. Acad. Sci. USA 2006, 103, 17143-17148. [PubMed]

10. Stewart, M.E.; Anderton, C.R.; Thompson, L.B.; Maria, J.; Gray, S.K.; Rogers, J.A.; Nuzzo, R.G. Nanostructured Plasmonic Sensors. Chem. Rev. 2008, 108, 494-521. [PubMed]

11. Stewart, M.E.; Yao, J.; Maria, J.; Gray, S.K.; Rogers, J.A.; Nuzzo, R.G. Multispectral Thin Film Biosensing and Quantitative Imaging Using 3D Plasmonic Crystals. Anal. Chem. 2009, 81, 5980-5989. [PubMed]

12. Yao, J.; Le, A.P.; Schulmerich, M.V.; Maria, J.; Lee, T.W.; Gray, S.K.; Bhargava, R.; Rogers, J.A.; Nuzzo, R.G. Soft Embossing of Nanoscale Optical and Plasmonic Structures in Glass. ACS Nano 2011, 5, 5763-5774. [CrossRef] [PubMed]

13. Baca, A.J.; Truong, T.T.; Cambrea, L.R.; Montgomery, J.M.; Gray, S.K.; Abdula, D.; Banks, T.R.; Yao, J.; Nuzzo, R.G.; Rogers, J.A. Molded plasmonic crystals for detecting and spatially imaging surface bound species by surface-enhanced Raman scattering. Appl. Phys. Lett. 2009, 94, 243109. [CrossRef] 
14. Baca, A.J.; Montgomery, J.M.; Cambrea, L.R.; Moran, M.; Johnson, L.; Yacoub, J.; Truong, T.T. Optimization of Nanopost Plasmonic Crystals for Surface Enhanced Raman Scattering. J. Phys. Chem. C 2011, 115, 7171-7178. [CrossRef]

15. Taflove, A.; Hagness, S.C. Computational Electrodynamics: The Finite-Difference Time-Domain Method; Artech House: London, UK, 2000.

16. Johnson, S.G.; Oskooi, A.; Taflove, A. (Eds.) Advances in FDTD Computational Electrodynamics: Photonics and Nanotechnology; Artech House: London, UK, 2013.

17. Mayer, K.M.; Hafner, J.H. Localized Surface Plasmon Resonance Sensors. Chem. Rev. 2011, 111, 3828-3857. [CrossRef] [PubMed]

18. Ungaro, C.; Gray, S.K.; Gupta, M.C. Graded-index structures for high-efficiency solar thermophotovoltaic emitting surfaces. Opt. Lett. 2014, 39, 5259-5262. [CrossRef] [PubMed]

19. Ono, A.; Kato, J.i.; Kawata, S. Subwavelength Optical Imaging through a Metallic Nanorod Array. Phys. Rev. Lett. 2005, 95, 267407. [CrossRef] [PubMed]

20. Zhang, Y.; Dou, X.; Dai, Y.; Wang, X.; Min, C.; Yuan, X. All-optical manipulation of micrometer-sized metallic particles. Photon. Res. 2018, 6, 66-71. [CrossRef]

21. Lu, H.; Gan, X.; Mao, D.; Jia, B.; Zhao, J. Flexibly tunable high-quality-factor induced transparency in plasmonic systems. Sci. Rep. 2018, 8, 1558. [CrossRef] [PubMed]

22. Oubre, C.; Nordlander, P. Finite-difference Time-domain Studies of the Optical Properties of Nanoshell Dimers. J. Phys. Chem. B 2005, 109, 10042-10051. [CrossRef] [PubMed]

23. Lal, S.; Grady, N.K.; Kundu, J.; Levin, C.S.; Lassiter, J.B.; Halas, N.J. Tailoring plasmonic substrates for surface enhanced spectroscopies. Chem. Soc. Rev. 2008, 37, 898-911. [CrossRef] [PubMed]

24. Wang, A.X.; Kong, X. Review of Recent Progress of Plasmonic Materials and Nano-Structures for SurfaceEnhanced Raman Scattering. Materials 2015, 8, 3024-3052. [CrossRef] [PubMed]

25. Grimault, A.S.; Vial, A.; de la Chapelle, M.L. Modeling of regular gold nanostructures arrays for SERS applications using a 3D FDTD method. Appl. Phys. B 2006, 84, 111-115. [CrossRef]

26. Baca, A.J.; Baca, J.; Montgomery, J.M.; Cambrea, L.R.; Funcheon, P.; Johnson, L.; Moran, M.; Connor, D. Mosaic-like Silver Nanobowl Plasmonic Crystals as Highly Active Surface-Enhanced Raman Scattering Substrates. J. Phys. Chem. 2015, 119, 17790-17799. [CrossRef]

27. Dhawan, A.; Gerhold, M.; Vo-Dinh, T. Theoretical Simulation and Focused Ion Beam Fabrication of Gold Nanostructures for Surface-Enhanced Raman Scattering (SERS). Nanobiotechnol 2007, 3, 164-171. [CrossRef] [PubMed]

28. Kang, S.; Lehman, S.E.; Schulmerich, M.V.; Le, A.P.; Lee, T.; Gray, S.K.; Bhargava, R.; Nuzzo, R.G. Refractive index sensing and surface-enhanced Raman spectroscopy using silver-gold layered bimetallic plasmonic crystals. Beilstein J. Nanotechnol. 2017, 8, 2492-2503. [CrossRef] [PubMed]

29. Haynes, C.L.; Van Duyne, R.P. Plasmon-Sampled Surface-Enhanced Raman Excitation Spectroscopy. J. Phys. Chem. B 2003, 107, 7426-7433. [CrossRef]

30. Ziegler, C.; Eychmüller, A. Seeded Growth Synthesis of Uniform Gold Nanoparticles with Diameters of 15-300 nm. J. Phys. Chem. C 2011, 115, 4502-4506. [CrossRef]

31. Oskooi, A.; Roundy, D.; Ibanescu, M.; Bermel, P.; Joannopoulos, J.; Johnson, S. MEEP: A flexible free-software package for electromagnetic simulations by the FDTD method. Comp. Phys. Comm. 2010, 181, 687-702. [CrossRef]

32. Johnston, B.; Christy, R. Optical constants of the noble metals. Phys. Rev. B 1972, 6, 4370. [CrossRef]

33. Oskooi, A.; Johnson, S. Distinguishing correct from incorrect PML proposals and a corrected unsplit PML for anisotropic, dispersive media. J. Comp. Phys. 2011, 230, 2369-2377. [CrossRef]

34. Mie, G. Beiträge zur Optik trüber Medien, speziell kolloidaler Metallösungen. Ann. Phys. 1908, 25, 377-445. [CrossRef]

35. Ahrens, J.; Geveci, B.; Law, C. ParaView: An End-User Tool for Large Data Visualization, Visualization Handbook; Elsevier: New York, NY, USA, 2005.

36. Ayachit, U. The ParaView Guide: A Parallel Visualization Application; Kitware: New York, NY, USA, 2015.

(c) 2018 by the authors. Licensee MDPI, Basel, Switzerland. This article is an open access article distributed under the terms and conditions of the Creative Commons Attribution (CC BY) license (http:/ / creativecommons.org/licenses/by/4.0/). 